\title{
KneTex - Improvements to a textile-integrated sensor system for feedback-supported rehabilitation after ACL surgery
}

Sinan Yavuz, Niederrhein University of Applied Sciences, Ambient Intelligence Laboratory, 47805 Krefeld, Germany, sinan.yavuz@hs-niederrhein.de

Nana Schlage, Lukas Cramer, Julia Demmer, Andreas Mühlen, Andreas Kitzig, Edwin Naroska and Gudrun Stockmanns, Niederrhein University of Applied Sciences, Ambient Intelligence Laboratory, 47805 Krefeld, Germany

\section{Introduction}

After ACL (anterior cruciate ligament) surgery, patients often experience an uncontrolled buckling of the knee ("giving way" effect). This and other effects, such as gait pattern errors, can have long-term consequences. Within the "KneTex" project, a system suitable for everyday use is being developed that uses textile-integrated sensors and actuators in a bandage to detect instability of the knee joint. To actively support the rehabilitation process, the user will be informed in the event of incorrect posture via vibration.

\section{Methods}

This abstract gives an overview of the current status of the Knetex project (hardware, software and signal processing).

The bandage system consists of two 9-axis IMUs (inertial measurement units) on the upper and lower leg, a barometric sensor, a resistive bending sensor and two vibration motors. The acquisition of the sensor data as well as the control of the actuators is carried out on a microcontroller board designed for the bandage system. The data is stored locally on a micro SD card and externally on a server.

Various functionalities of the bandage system can be executed (labeling data, storing data on the server, controlling the actuators etc.) via a custom app which is developed as part of the project. The sensor values are sent encrypted to the Knetex server, where they can be further processed and evaluated by an authorised physiotherapist/physician for further therapy.

Based on the IMU data, flexion/extension, rotation and varus/valgus are calculated using an adapted Rauch-TungStriebel approach. The recognition system is based on a random forest classifier, which is trained by pre-labeled sensor data and currently allows the classification of the motion patterns "walking", "lying", "sitting", "standing" and "up/down stairs" and can be extended by new models.

\section{Results and Conclusion}

With the Knetex system movement data (actions/postures and knee angle) can currently recorded, stored and processed locally as well as on a server. The data is already labeled so the individual movement data can be directly correlated with the action performed. Currently, investigations are being carried out for the calibration of the IMUs to optimize the calculation of the knee angles. The recognition system enables a reliable classification of the movement patterns with high accuracy. Tests with the bandage system in our motion laboratory already show good results in comparison with the Qualisys reference system. Furthermore, the data will be used to be able to automatically control the actuators via defined threshold values of the angles etc. 


\section{Evaluation of an algorithm for optical pulse detection in children for application on the Pepper robot}

Nina Goes, Fraunhofer IIS and FAU Erlangen-Nürnberg, Germany, nina.goes@fau.de Julian Sessner, Institute for Factory Automation and Production System, FAU Erlangen-Nürnberg, Germany, julian.sessner@faps.fau.de

Isabel Dziobek, Clinical Psychology of Social Interaction, HU Berlin, Berlin, Germany, isabel.dziobek@hu-berlin.de Jan Steffan, Fraunhofer IIS, Erlangen, Germany, jan.steffan @iis.fraunhofer.de Matthias Struck, Fraunhofer IIS, Erlangen, Germany, matthias.struck@iis.fraunhofer.de Jörg Franke, Institute for Factory Automation and Production System, FAU Erlangen-Nürnberg, Germany, joerg.franke@faps.fau.de

Simone Kirst, Clinical Psychology of Social Interaction, HU Berlin, Germany, simone.kirst@hu-berlin.de Sandra Naumann, Clinical Psychology of Social Interaction, HU Berlin, Germany, sandra.naumann@hu-berlin.de Thomas Wittenberg, Fraunhofer IIS and FAU Erlangen-Nürnberg, Germany, thomas.wittenberg@iis.fraunhofer.de Nadine Lang, Fraunhofer IIS, Erlangen, Germany, nadine.lang@iis.fraunhofer.de

\section{Introduction}

In order to engage in socio-emotional interactions, children with autism spectrum conditions need support to understand and convey emotions. In our approach, a humanoid robot (Pepper) is incorporated acting as a tutor for the child and thus supports the therapist. The robot, equipped with multimodal sensor technology to acquire the emotional feedback of the child, acts as a tutor for the child asking to perform tasks, adapted to the current arousal state. By increasing or decreasing the difficulties of implemented therapy modules, the child can be given the appropriate task according to its emotional state.

\section{Methods}

The child's arousal is measured with different techniques implemented in and on the robot: emotion detection based on audio recordings of the speech signal and camera detected facial expressions or pulse rate. To this end, the remote Photoplethysmography (rPPG) signal from camera recordings of the subjects' face is acquired. While its unintrusive measurement is a huge advantage, the major drawback for $\mathrm{PPPG}$ is its proneness to motion and light artefacts generally requiring signal de-noising steps. We implemented a wavelet transform based on the log-Gabor wavelets and a real-time filter bank with 32 filters. The signal was filtered with a prior filter and afterward with a Markov chain in order to extract the underlying pulse rate.

\section{Results}

Within an initial study, $n=5$ children were observed, watching videos with different connotated emotions. As a reference for the heart rate (HR), a wristband (E4 empatica) was used. The captured emotions of all subjects were manually annotated to identify low and high arousal parts and positive and negative emotions. The automatically extracted heart rates from the rPPG-data indicated a correlation with annotated emotions.

\section{Conclusions}

Extracting the HR from children's rPPG signals is challenging as those signals are prone to artefacts. Nevertheless, by adequately filtering and pre-processing the raw rPPG signal, a robust extraction of the HR is possible. Furthermore, the additional information can help therapists interpreting the socio-emotional interactions and enhance the usage of robots as therapy support. 


\section{Development of a mobile, cost-effective and easy to use inertial motion capture system for monitoring in rehabilitation applications}

Nana Schlage, Niederrhein University of Applied Sciences, Ambient Intelligence Laboratoy, 47805 Krefeld, Germany, nana.schlage@hs-niederrhein.de Andreas Kitzig, Gudrun Stockmanns, Edwin Naroska, Niederrhein University of Applied Sciences, Ambient Intelligence Laboratoy, 47805 Krefeld, Germany

\section{Introduction}

The scope of motion capturing (MoCap) is becoming more extensive and is thus expanding into various areas of applications including the entertainment sector, such as film making, game design, and also medicine and sport science to support diagnosis as well as to optimise performance of athletes.

Most marker-based or mechanical MoCap systems have different limitations with respect to the number of test persons that can be captured concurrently, are often very expensive, being limited in the type of movements that are supported due to the used technology or are restricted to a certain location.

To circumvent these limitations, movements can also be captured with cost-effective inertial measurement units (IMU) based development systems. Examples for those systems are KineXYZ or Notch. However, these systems only focus on certain applications and can only be used with the intended sensors. In order to overcome these limitations, this abstract presents a mobile, easy-to-use and easily expandable variation of a motion capture system for the lower extremities, implemented using a low-cost IMU-based development system.

\section{Methods}

The presented MoCap system can be used stand-alone or in addition as a reference for systems under development in the field of research and development of rehabilitation applications such as gait analysis. The mobile MoCap system focuses on applications to capture human movements and to derive joint angles of the lower extremities. It includes a total of five commercially available IMU Nodes (XSens Dots) attachted to the body with elastic straps and a specially developed application for a mobile device.

To use the system, the sensors must be placed on the lower and upper thighs and on the lower back. Then, the five sensors are connected, synchronized and calibrated with the smartphone. Thereafter, the system is ready for operation and acquisition and further processing of the sensor data can be started.

The authors focus development on biosignal processing as well as realization of a model of the lower extrimities and are currently extending the overall system as a reference measurement system in a research project for the derivation of malpositions of the human knee. The system can also be extended to other body regions due to its modular design. The different knee angles are calculated from the orientations of the pairs of sensors, each of which is located together on one leg. In addition, with the help of a simple kinematic chain, a stickman model of the respective person can be created and displayed. A marker-based system (Qualisys) is used for evaluation.

\section{Results and Conclusion}

The presented system is a low-cost, mobile variant of a MoCap system suitable for rehabilitation applications and sports but is also usable as a reference for research and development. It may not be as accurate as expensive systems due to the small number of sensors used, so it should be seen as an addition rather than a replacement, but it has a high usability due to its fast and uncomplicated installation in the field. Furthermore, it is easily expandable, which means that it can be easily adapted to different applications. 


\title{
"Pandemic Robot" - A concept for robot-based surveillance of inhabitants in elderly and nursing homes during pandemic situations
}

\author{
Jochen Bauer, Friedrich-Alexander-Universität Erlangen-Nuremberg, Germany, jochen.bauer@faps.fau.de \\ Bruno Ristok, C+S AG, Augsburg, Germany, brunoristok@,cs-ag.de \\ Simon Dengler, Friedrich-Alexander-Universität Erlangen-Nuremberg, Germany, simon.dengler@faps.fau.de \\ Jörg Franke, , Friedrich-Alexander-Universität Erlangen-Nuremberg, Germany, franke@,faps.fau.de \\ Simon Weigele, C+S AG, Augsburg, Germany, simonweigele@cs-ag.de \\ Matthias Struck, Fraunhofer IIS, Erlangen, Germany, matthias,struck@iiis.fraunhofer.de \\ Robert Frischholz, BioId GmbH, Nuremberg, Germany, r.frischholz@,bioid.com \\ Thomas Wittenberg, Fraunhofer IIS, Erlangen, Germany, thomas.wittenberg@iiis.fraunhofer.de
}

\section{Introduction}

Robot-based service platforms are currently establishing themselves as new and affordable variants for supporting care in elderly-, retirement and nursing homes. Many of these are open multifunctional platforms, which can potentially be integrated into a variety of such environments, if the necessary WIFI infrastructure is available. Furthermore, many personalized services can be realized offered on these platforms which can be used to foster distant interactions between inhabitants and care-provider, while simultaneously keeping up the quality of life of the inhabitants.

Within this contribution we will sketch such a combination of technologies to be used for caregiving and health monitoring during pandemic situations.

\section{Methods}

Partially open mobile robotic platforms such as the Temi, Sanbot, Pepper, or others allow the extension with adequate new sensors. In order to detect infectious diseases of patients, residents, relatives or healthcareprofessionals, a meaningful set of contactless, optical sensors would include the assessment of vital-signs such as heartrate and heartrate-variability, respiratory-rate, blood-oxygen-saturation and temperature. Additionally, demographic data (age, gender, constitution) of the observed person is needed, which can either be acquired by communication with the electronic patient record or alternatively obtained by facial image analysis. As these multifunctional platforms are usually capable for telepresence, in case of detecting an infected person, these systems support video conferencing with their built-in cameras and microphones, the system can independently plan and initiate teleconsultations with a physician. Finally, the interaction with the electronic care record is essential, to upload acquired vital data and emotional information as well download essential background information.

\section{Results}

Almost all of the named technologies (contactless optical sensors, image based facial analysis, mobile telepresence and video-conference systems, electronic care records, ... ) have been under strong investigation in the past years and are currently moving from laboratory settings to "in-the-wild" scenarios. Nevertheless, the smooth integration of all necessary components into one common software-architecture using adequate standardized softwareinterfaces, their bilateral interaction as well the data fusion and the (AI-based) data analysis are still open points to be addressed and tackled in the near future.

\section{Conclusion}

In support the distancing between patients and care-providers during pandemic situations, while maintaining the quality of life, new technologies and infrastructures are necessary, which are partially available but there is some additional need for further integrations and improved interoperability. 


\title{
To improve patients' empowerment by self-connecting in home parenteral nutrition
}

\author{
Völz Diana*, Eisele Joschua*, Benderoth Günther*, Schulze Ulrike** \\ D Frankfurt Univerity of Applied Sciences, Faculty of Coomputer Science and Engineering*, Faculty of Health \\ and Social Work* \\ voelz@fb2.fra-uas.de
}

\section{Introduction}

One promising approach for patients needing parenteral nutrition to live an almost normal live is to provide extra-hospital therapy supported by an ambulatory care service who connect and disconnect the nutritional fluid day by day. The nutrition therapy takes between 12 to 16 hours every day. Studies show that this therapy disturbs patients' all day life. Aim of this project is to enable a more autonomous life for patients by self-determined in between disconnecting and connection of the therapy. However, any interruption of the nutrition process poses health risks to the patient, if air bubbles or biocontaminants enter the tube system.

\section{Methods}

Analysing expert talks, conducted by cooperating nursing academics, impact factors were dedected to be used to develop prototyps of new manageable connecting systems between tube and port catheter, which are afterwards the basis in an experimental setup. The operation with those connection systems are part of an participative observation with selected participants group. The influence parameters of the study are age, handicap and professional qualification. The target parameters are leaking, successfully connecting and disconnecting, ergonomics and connecting time. Subsequently a standardised survey is carried out with the participants which is quantatively and qualitatively analysed.

\section{Results}

Results show the quantitative relations of the influencing parameters and detects additional requirements and wishes of the participants. These results and the requirements addressed by the expert talks are the starting point for redesigning the connecting system.

\section{Conclusion}

The research project "EasyGoing@Home", funded by Universities of Applied Sciences in Hesse and in cooperation with Fresenius Kabi Deutschland $\mathrm{GmbH}$, takes one step forward in enabling empowerment for patients by self-connecting in home parenteral nutrition due to redesigning the closure of the tube system to the port catheter. In a follow-up project, the automated flushing with saline solution through a pump will be evolved. 


\section{Development of an assistive mobile app to improve informal home care}

Tom Jürgens, Institute for Biomedical Engineering (IBMT), FB Life Science Engineering (LSE), Technische Hochschule Mittelhessen (THM) - University of Applied Sciences, Gießen, Germany, tom.juergens@1se.thm.de

Pavel Larionov, Institute for Biomedical Engineering (IBMT), FB Life Science Engineering (LSE), Technische Hochschule Mittelhessen (THM) - University of Applied Sciences, Gießen, Germany, pavel.larionov@1se.thm.de

Wolfgang George, FB Management und Kommunikation (MuK), Technische Hochschule Mittelhessen (THM) University of Applied Sciences, Gießen, Germany, wolfgang.george@muk.thm.de

Thomas Schanze, Institute for Biomedical Engineering (IBMT), FB Life Science Engineering (LSE), Technische Hochschule Mittelhessen (THM) - University of Applied Sciences, Gießen, Germany, thomas.schanze@1se.thm.de

\section{Introduction}

Informal home and subsequent palliative care are highly sensitive and require broad knowledge. In this context, the right information at the right time and a solid health competence, is crucial. The paper describes a digital health application (DiGA) concept that is intended to support the caregiver to provide informal care and the affected persons.

\section{Methods}

The iterative and agile development process is performed in close collaboration with palliative care, advanced care and scientific experts. This allowed to determine the main application purpose, delivering appropriate information as well as creating a high-fidelity prototype with Adobe XD. Following agile principles and in order to minimize adjustment costs, first tests with field experts were performed with the prototype. Based this high-fidelity prototype, the implementation for iOS is performed with the IDE Xcode and UIKit. For the Android OS the Flutter SDK is used.

\section{Results}

The main application functionalities are divided into four hubs: for increasing knowledge and competence (Knowledge $\mathrm{Hub}$ ), to organize communication (Contacts Hub), to store and manage data (Data Hub) and to overview the whole process (Home Hub). The Knowledge Hub, as the current main value-adding feature, provides empowerment to the caregivers by enhancing the competence via bite-sized mobile friendly formatted articles. The Data and the Contacts Hub allow to store person-specific data and documents as well as care-specific contacts. And the Home Hub allows users to navigate in the app via intelligent situation-specific suggestions and reading-lists. Overall, the caregiver application aims to increase user awareness and competence, simplifying the decision-making and building foundations for a user-centric, crossinstitutional case management system.

\section{Conclusion}

As a user-empowering solution for informal home and palliative care, it is highly likely that the app relieves the caregiver and the affected person by increasing the health literacy and providing functions for simple and transparent management of the care situation. Furthermore, it enables the Caregiver to act increasingly as a case manager, minimizing risks of misunderstanding between the stakeholders and increase the quality of life for all persons involved. Based on the obtained functionalities, a so-called mobile medical app (DiGA) can be developed.

Sponsored by The Federal Joint Committee of Germany (G-BA, grant 01VSF17044). 


\section{Virtual Therapeutics - Use Case Requirements to deliver value with Virtual Reality and Biofeedback Applications for alcohol addiction therapy}

Christian Hanshans*, Department of Applied Sciences and Mechatronics, University of Applied Science Munich, Munich, christian.hanshans@hm.edu

Bettina Maisch, Strascheg Center for Entrepreneurship, Department of Qualification and Education, University of Applied Science Munich, Munich, bettina.maisch@sce.de

Johannes Zauner Department of Applied Sciences and Mechatronics, University of Applied Science Munich, Munich, johannes.zauner@hm.edu

Moritz M. R. Faust, Department of Applied Sciences and Mechatronics, University of Applied Science Munich, Munich, faust@hm.edu

Lukas M. Bröll, Department of Applied Sciences and Mechatronics, University of Applied Science Munich, Munich, 1.broell@hm.edu

Susanne Karch, Department of Psychiatry and Psychotherapy, University Hospital LMU, Munich, Susanne.Karch@med.uni-muenchen.de

* corresponding author

\section{Introduction}

The application of virtual reality (VR) as a supportive tool in psychotherapy has gained great popularity in recent years. The almost unlimited possibilities of immersion in computer-generated VR environments allow practitioners to interactively and realistically simulate certain scenarios or train patients with the help of animations or interacting with an artificial environment.

\section{Methods}

Besides literature research, we conducted an analysis of existing technical solutions in the field of virtual addiction treatment as well as clinical requirement of therapists. Based on our analysis we selected several easy to use and costeffective VR headsets, identified a shopping environment as the most promising exposition scenario and HRV analysis as the best choice for measuring craving.

\section{Results}

For further patient studies, a virtual shopping scenario and an easy to use hardware setup consisting of a common gaming VR headset and an HRV measuring system, that can be integrated in the simulation has been developed. The focus of the development was set to the demands of clinical settings. Through our proof of concept, we demonstrated that virtual therapy can be implemented for reasonable costs and can support the treatment with additional physiological data needed to parameterize and objectify the state-of-the art treatment by recording physiological reactions to offered stimuli. This brings added value for patients (psychoeducation) and treatment providers (progress documentation). We uncovered that automatic interaction of the simulation (increase or decrease of the stimulus intensity or change into a coping scenario) on physiological changes would be a clear increase of the value in the treatment process.

\section{Conclusion}

Virtual Reality has the potential to enhance the treatment of alcohol addiction. The combination of software and hardware, that seamlessly integrates in the current therapeutical approach, lays the foundation for upcoming clinical use and trials to proof the better outcome of VR enhanced addiction therapy. 


\section{Traditional versus Neural Network Classification Methods for Facial Emotion Recognition}

Herag Arabian, Institute for Technical Medicine (ITeM), Hochschule Furtwangen University, 78054 VS-Schwenningen, Germany, H.Arabian@hs-furtwangen.de

Verena Wagner-Hartl, Department of Industrial Technologies, Hochshule Furtwangen University, 78532 Tuttlingen, Germany

Knut Möller, Institute for Technical Medicine (ITeM), Hochschule Furtwangen University, 78054 VS-Schwenningen, Germany

\section{Introduction}

A feedback system for the emotional training of children with Autism Spectrum Disorder is being developed. One of the system components is devoted to the recognition of emotions from facial expressions. The aim of this study is to show the effectiveness between implementing traditional machine learning techniques with feature extraction and the implementation of a Deep Neural Network for classification purposes in order to find a Facial Emotion Recognition (FER) model that is suitable to be implemented in a closed loop real time system.

\section{Methods}

Local regions of interest, highlighting the facial component, within an image captured by a webcam during an educative virtual game is tested for FER. The recognition classification model using Machine Learning techniques based on KNearest Neighbor, Support Vector Machine, and Transfer Learning using the popular AlexNet architecture was implemented. The performance of the models were based on statistical data analysis of classification accuracies from varying randomly selected training and validation sets of the Oulu-CASIA database.

\section{Results}

Accuracies of up to $98.94 \%$ for traditional, and $98.64 \%$ for the network, with small variations in data distribution were observed. When testing the models against images from subjects not found in the Oulu-CASIA database, the models showed better robustness at emotion classes of Happiness and Surprise.

\section{Conclusion}

The statistical data showed that both methods were able to achieve similar accuracy levels. The traditional method provides an effective compromise between accuracy, number of extracted features, speed and computational costs. Further research is required to analyse the regions that the network designates as important for classification. 